\title{
Anorectal Malformation with Colonic Duplication and Dual Rectovaginal Fistulae
}

Platt $\mathrm{KD}^{1}$, Wu AJ ${ }^{1}$, Nunez Lopez $\mathrm{O}^{2}$, Wang $\mathrm{KS}^{1,3}$, Bowen-Jallow $\mathrm{KA}^{2^{*}}$

${ }^{1}$ Keck School of Medicine of the University of Southern California, Los Angeles, CA.

${ }^{2}$ University of Texas Medical Branch, Galveston, TX.

${ }^{3}$ Division of Pediatric Surgery, Children's Hospital Los Angeles, Los Angeles, CA.

\section{Abstract}

Anorectal malformations associated with colonic duplications are rare. We report a case of imperforate anus, tubular colorectal duplication, and dual rectovaginal fistulae. This case illustrates the diagnostic challenge and variable presentation of colonic duplications and the use of laparoscopy in the surgical management. A review of the etiology, presentation, and surgical therapy is presented.

\section{Introduction}

Colonic duplications are uncommon congenital anomalies with widely varied presentations. Associated genitourinary anomalies can frequently be found in patients with hindgut duplications. The association with anorectal malformations is well described, but the involvement of the colon is rare. The broad spectrum of coexisting anatomical abnormalities represent a diagnostic and therapeutic challenge.

\section{Case Description}

A 7-month-old girl with a history of imperforate anus, status post diverting colostomy and mucous fistula creation at birth, was referred to our center for further evaluation and treatment. The girl had a history of cleft lip and palate repair, small ventricular septal defect, and hydroureter. Physical examination revealed an anorectal malformation without perineal opening. A distal colostogram demonstrated a rectovaginal fistula (RVF). Rectovaginal fistulae in the setting of anorectal malformations are rare. To confirm this finding, a 15-french rubber catheter was advanced through the mucus fistula and observed exiting from the vagina. Endoscopic vaginoscopy demonstrated a vaginal septum and the RVF as evidenced by the rubber catheter. The vaginal septum was excised, the fistula was divided, and a posterior sagittal anorectoplasty procedure (PSARP) was performed.
After four months, the colostomy and mucous fistula were taken down uneventfully. However, three weeks later the patient presented with stool drainage from her vagina, causing concern for a recurrent RVF. A distal colostogram (Figures 1A, 1B) and a barium enema were performed demonstrating a rectovaginal fistula. The diverting colostomy and mucus fistula were reestablished.

At 18 months of age, the patient was taken back to the operating room for RVF repair. The patient was placed in the prone position and using the prior PSARP incision the posterior rectal wall was split. No fistula was found despite meticulous examination of the anterior rectal wall. The patient was then placed in the supine position and three 3 -mm-trocars were used for laparoscopic exploration. Two ovaries with associated fallopian tubes were observed, but only an atretic uterine cord was found. Distal to the mucous fistula, we found a single colonic lumen bifurcated into two lumens. One colonic lumen led to the rectum and one colonic lumen led to the vagina consistent with a colorectal duplication (Figure 2). Closer examination of the preoperative barium enema, revealed one fluoroscopic image of a colonic common wall consistent with tubular colonic duplication (TCD) (Figure 3).

Laparoscopically, the colonic duplication leading to the vagina was divided using an Endo GIA stapler (Figure 4). Then, under laparoscopic guidance, the Endo GIA stapler was advanced through the mucous fistula and the common wall of the colonic

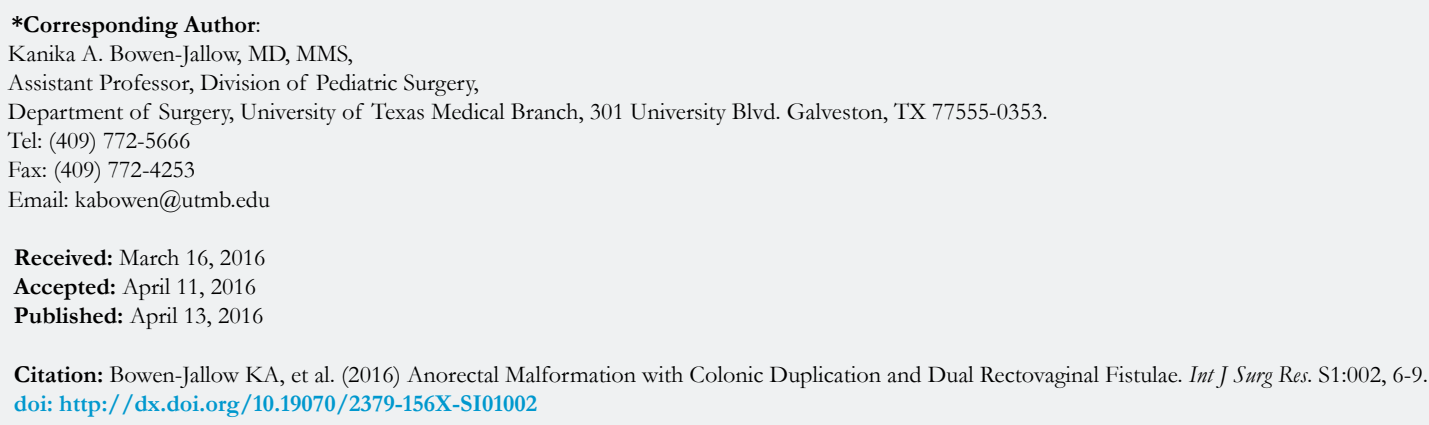

Copyright: Bowen-Jallow KA ${ }^{\circ}$ 2016. This is an open-access article distributed under the terms of the Creative Commons Attribution License, which permits unrestricted use, distribution and reproduction in any medium, provided the original author and source are credited. 
Figure 1. Distal colostogram demonstrating a rectovaginal fistula (white arrow).

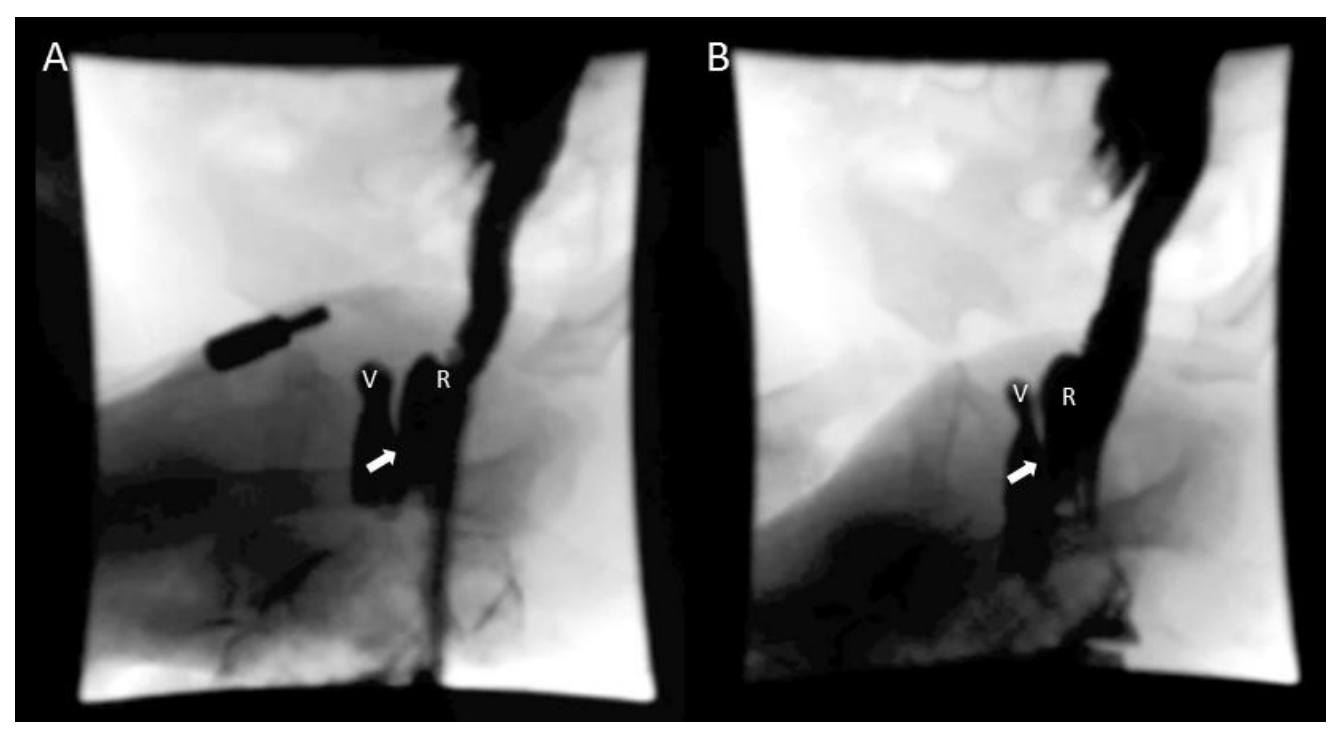

Figure 2. Tubular colonic duplication with one colonic lumen (A) leading to the rectum and one colonic lumen (B) leading to the vagina.

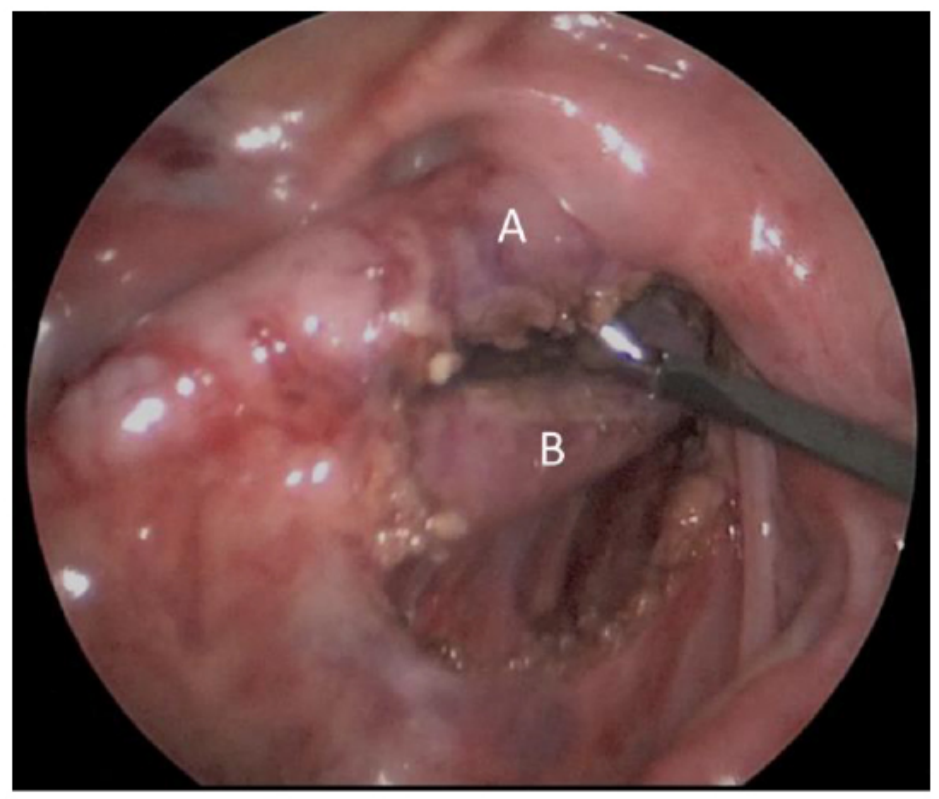

Figure 3. Fluoroscopic image demonstrating a single colonic lumen (arrowhead) that bifurcates into a tubular colonic duplication with a common wall (white arrow).

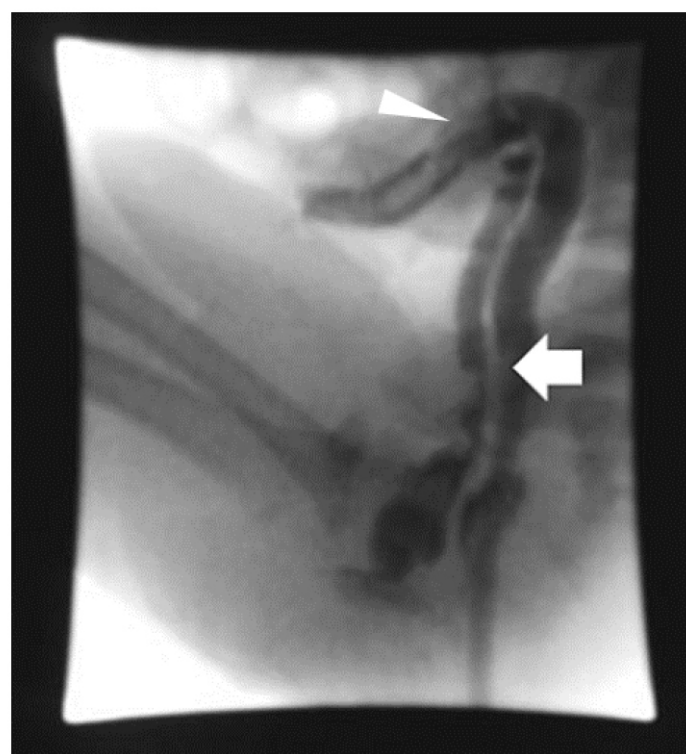


Figure 4. Laparoscopic image after division of the duplicated colon (B) from the vagina (V). Posteriorly, the colonic lumen leading to the rectum can be appreciated (A).

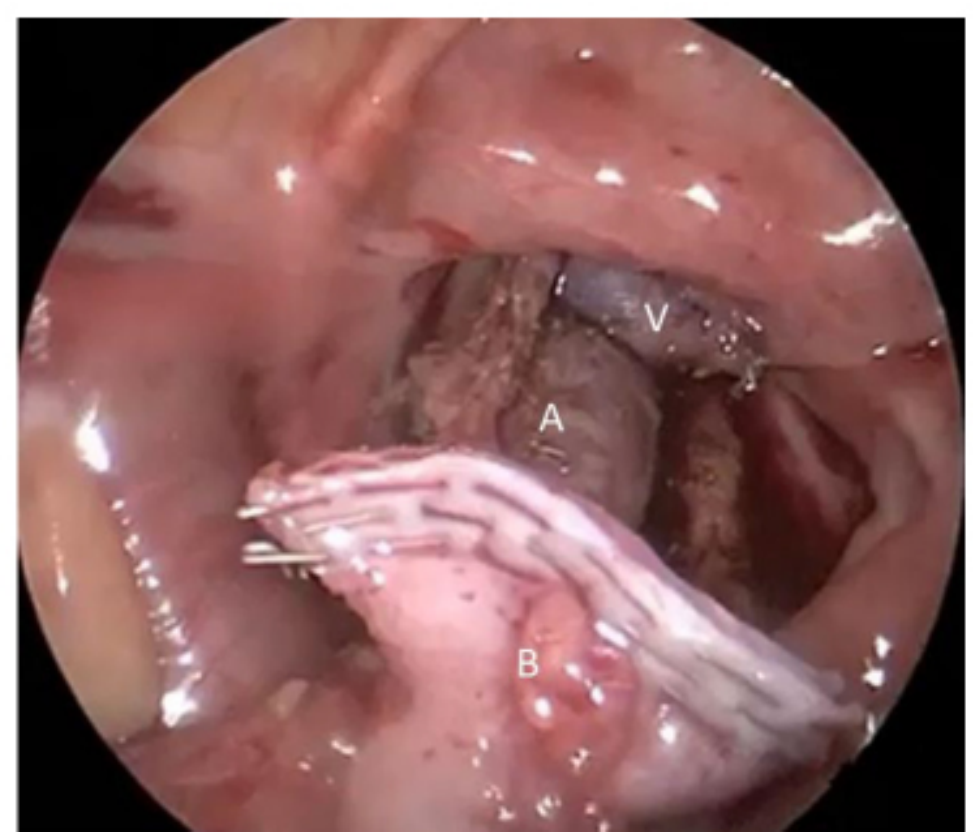

Figure 5. Endo GIA stapler advanced through the mucous fistula under laparoscopic observation for division of the common wall of the colonic duplication.

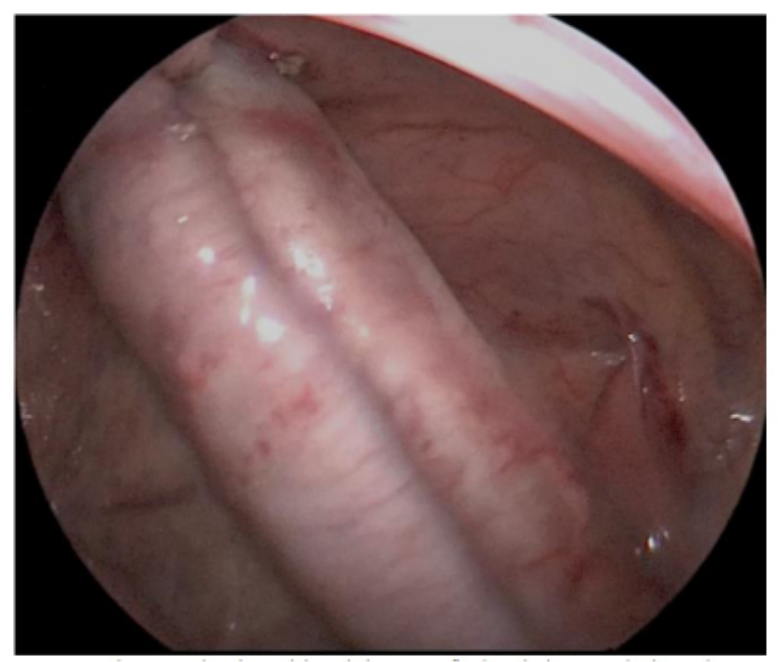

duplication was divided endoluminally, thereby creating a single lumen (Figure 5). An air leak test was performed without evidence of leak. The patient was placed back into the prone position to complete the redo PSARP.

Three months later, the patient had a distal colostogram that was normal and underwent a colostomy takedown without complications. Two years later, the patient is doing well, stooling per anus and free from vaginal drainage.

\section{Discussion}

Gastrointestinal tract duplications are rare congenital anomalies, with a reported incidence of one in 4,500 life births [1,2]. Duplications can occur anywhere along the alimentary tract from the mouth to the anus, most commonly affecting the ileum (33$60 \%$ ) $[2,3]$. Approximately $80 \%$ of the duplications are cystic, without communication to the adjacent gastrointestinal tract, the remaining are tubular, and communicate with the intestinal lumen [3, 4]. Tubular colonic duplications are associated with genitourinary malformations including duplication of genitourinary structures, as well as fistulae between the duplicated colon and the genitourinary system or perineum. The association with anorectal malformations is also well established [5].

Several theories have been proposed to explain the embryologic etiology of gastrointestinal duplications; however, no single hypothesis can explain all duplications and their associated anomalies $[3,6]$. Tubular duplications of the hindgut as seen in this case, associated with genitourinary abnormalities and anorectal malformations, can be explained by an early abnormal development of the caudal cell mass during the $23^{\text {rd }}$ to $25^{\text {th }}$ day of gestation. Formation of aberrant fibrous bands can divide the adjacent mesoderm causing duplication of the gastrointestinal tract and septation or duplication of the genitourinary tract $[7,8]$.

Gastrointestinal duplications have variable clinical presentations depending on the size, location, presence of ectopic mucosa, and 
association with a fistula. Duplications can be diagnosed prenatally or found before symptoms develop in patients undergoing workup for associated malformations [4, 9]. Symptoms vary greatly and may include abdominal mass, abdominal pain, bowel obstruction, intestinal gangrene due to compression of the vasculature, gastrointestinal bleeding from ulceration of ectopic mucosa, perforation secondary to ulceration, volvulus, or intussusception $[2,3,6]$.

The patient in this case presented with a tubular colorectal duplication (TCD), which is among the rarest of gastrointestinal duplications, accounting for an estimated $6 \%$ of cases [5]. Additional anomalies of other systems, as seen in this child, are present in more than three quarters of patients with TCD $[5,10]$.

The child presented in this report, had a TCD with associated anorectal malformation and two rectovaginal fistulae. However, the diagnosis of TCD was initially missed. At first, a single RVF was diagnosed preoperatively with a distal colostogram, which was divided and repaired during the initial PSARP. The second RVF, thought to be a recurrence of the previously repaired fistula, was indeed a different fistula between the duplicated bowel and the vagina. The colonic duplication ending to the vagina was diagnosed by laparoscopy.

Because gastrointestinal duplications can present with a wide range of clinical manifestations or may be found intraoperatively, as in this case, proper surgical management requires an experienced pediatric surgeon familiar with the anatomy and clinical characteristic of these lesions. The treatment is surgical in all the cases in order to treat or prevent complications $[1,4,6]$.

Laparoscopic treatment of gastrointestinal duplications is well documented. Esophageal duplications, gastric and duodenal duplication cysts, and thoraco-abdominal enteral duplication cysts have been successfully treated using laparoscopy. Main techniques for duplications of the foregut and midgut include cyst excision, cyst unroofing, cyst fulguration, and resection $[4,11,12]$.

In hindgut tubular duplications, the precise anatomy needs to be determined in order to define the operative plan. Definitive surgery consists of disconnection of the fistula associated with the colon using the standard approach of posterior sagittal reconstruction and anoplasty. If present, a fistula associated with the duplicated bowel is also disconnected. The duplicated bowel, especially when its length is significant, should be preserved to optimize the functional outcome of the anorectal reconstruction. Endoscopic section of the common wall is a valid approach when preserving the duplicated bowel, eliminating the existence of a blind pouch and the risk of future complications associated with it $[1,13]$.

In summary, a tubular colonic duplication associated with anorectal malformation and dual rectovaginal fistulae is rare. The preoperative diagnosis is challenging and surgical correction is mandatory and must be individualized. In carefully selected patients, a posterior sagittal approach in combination with laparoscopy is the best alternative in the cases that require abdominal exploration.

\section{References}

[1]. Holcomb GW, Gheissari A, O'Neill JA, Shorter NA, Bishop HC (1989) Surgical management of alimentary tract duplications. Ann Surg 209(2): 167-174.

[2]. Puligandla PS, Nguyen LT, St-Vil D, Flageole H, Bensoussan AL, et al. (2003) Gastrointestinal duplications. J Pediatr Surg 38(5): 740-744.

[3]. Macpherson RI (1993) Gastrointestinal tract duplications: clinical, pathologic, etiologic, and radiologic considerations. Radiographics 13(5): 10631080.

[4]. Mayer JP, Bettolli M (2014) Alimentary tract duplications in newborns and children: Diagnostic aspects and the role of laparoscopic treatment. World J Gastroenterol 20(39): 14263-14271.

[5]. Yousefzadeh DK, Bickers GH, Jackson JH Jr, Benton C (1983) Tubular colonic duplication- review of 1876-1981 literature. Pediatr Radiol 13(2): 65-71.

[6]. Iyer CP, Mahour GH (1995) Duplications of the alimentary tract in infants and children. J Pediatr Surg 30(9): 1267-1270.

[7]. Dominguez R, Rott J, Castillo M, Pittaluga RR, Corriere N Jr (1993) Caudal Duplication Syndrome. Am J Dis Child 147(10): 1048-1052.

[8]. Siebert JR, Rutledge JC, Kapur RP (2005) Association of cloacal anomalies, caudal duplication, and twinning. Pediatr Dev Pathol 8(3): 339-354.

[9]. Laje P, Flake AW, Adzick NS (2010) Prenatal diagnosis and postnatal resection of intraabdominal enteric duplications. J Pediatr Surg 45(7): 15541558.

[10]. Sengar M, Gupta CR, Jain V, Mohta A (2013) Colorectal duplication with prostatorectal fistulae. J Pediatr Surg 48(4): 869-872.

[11]. Pujar VC, Kurbet S, Kaltari DK (2013) Laparoscopic excision of intra-abdominal oesophageal duplication cyst in a child. J Minim Access Surg 9(1): 34-36.

[12]. Schleef J, Schalamon J (2000) The role of laparoscopy in the diagnosis and treatment of intestinal duplication in childhood. A report of two cases. Surg Endosc 14(9): 865.

[13]. Craigie RJ, Abbaraju JS, Ba’ath ME, Turnock RR, Baillie CT (2006) Anorectal malformation with tubular hindgut duplication. J Pediatr Surg 41(6): e31-34.

Special Issue on
"Neonatal and Pediatric Surgery"
Edited by:
Prakash Kabbur,
Kapiolani Medical Center for Women and Children
Honolulu, USA
E-mail: kabburpm@yahoo.com

